Reprinted from the Proceedings of the Cambridge Philosophical Society,

Volume 51, Part 4, pp. 551-553, 1955.

PRINTED IN GREAT BRTTATN

\title{
PAIRS OF MATRICES WITH A NON-ZERO COMMUTATOR
}

\author{
BY L. S. GODDARD AND H. SCHNEIDER
}

\section{Received 21 January 1955}

1. This note takes its origin in a remark by Brauer(1) and Perfect(5): Let $A$ be a square complex matrix of order $n$ whose characteristic roots are $\alpha_{1}, \ldots, \alpha_{n}$. If $\mathbf{x}_{1}$ is a characteristic column vector with associated root $\alpha_{1}$ and $k$ is any row vector, then the characteristic roots of $A+\mathbf{x}_{1} \mathbf{k}$ are $\alpha_{1}+\mathbf{k x}_{1}, \alpha_{2}, \ldots, \alpha_{n}$. Recently, Goddard (2) extended this result as follows: If $\mathbf{x}_{1}, \ldots, \mathbf{x}_{r}$ are linearly independent characteristic column vectors associated with the characteristic roots $\alpha_{1}, \ldots, \alpha_{r}$ of the matrix $A$, whose elements lie in any algebraically closed field, then any characteristic root of $\Lambda+K X$ is also a characteristic root of $A+X K$, where $K$ is an arbitrary $r \times n$ matrix, $X=\left(x_{1}, \ldots, x_{p}\right)$ and $\Lambda=\operatorname{diag}\left(\alpha_{1}, \ldots, \alpha_{r}\right)$. We shall prove some theorems of which these and other well-known results are special cases.

2. Let $F$ be an arbitrary field. By $f(x, y)$ we shall denote a polynomial, with coefficients in $F$, in two non-commutative indeterminates $x$ and $y$, and we shall set $f_{0}(x)=f(x, 0)$. By $F_{n}$ we shall mean the ring of square matrices of order $n$ with elements in $F$. In order to a void exceptional cases, we adopt the convention that the characteristic polynomial of the empty matrix (the $0 \times 0$ matrix) is unity.

Let $A$ and $B$ be matrices in $F_{n}$ and $F_{m}$ respectively. By a commutator from $A$ to $B$ we shall mean an $n \times m$ matrix $X$, with elements in $F$, satisfying $A X=X B$. If $X$ is a commutator from $A$ to $B$ then $X$ also satisfies $f(A, X K) X=X f(B, K X)$, where $K$ is any $m \times n$ matrix with elements in $F$. We shall prove the following theorem.

Theorem 1. Let $A$ and $B$ be matrices in $F_{n}$ and $F_{m}$ respectively and let $X$ be a commutator from $A$ to $B$ of rank $r$. Then, for any polynomial $f(x, y)$ and any $m \times n$ matrix $K$, with elements in $F$,

$$
\begin{aligned}
& \left|\lambda I_{n}-f(A, X K)\right|=\theta(\lambda) p(\lambda), \\
& \left|\lambda I_{m}-f(B, K X)\right|=\theta(\lambda) q(\lambda),
\end{aligned}
$$

where (i) $\theta(\lambda), p(\lambda), q(\lambda)$ are polynomials in $F[\lambda]$ of degrees $r, n-r, m-r$, respectively, (ii) $p(\lambda), q(\lambda)$, are independent of $K$ and are therefore factors of the characteristic polynomials of $f_{0}(A)$ and $f_{0}(B)$ respectively.

Proof. If $r=0$ the result is trivial. If $r=\min (n, m)$ then either $\dot{p}(\lambda)$ or $q(\lambda)$ is unity, and it is easily seen that the proof given below for the case $0<r<\min (n, m)$ is still valid, provided that some of the submatrices of the partitioned matrices which occur are omitted.

Let $P$ and $Q$ be non-singular matrices in $F_{n}$ and $F_{m}$ respectively for which

$$
P \not{X} Q^{-1}=\left(\begin{array}{cc}
I_{\vec{r}} & 0 \\
0 & 0
\end{array}\right)=Y \text {, say. }
$$


Property $P_{n}$ is then property $P$ (cf. McCoy (3)). Theorem 1 implies that the pairs of matrices $A, X K$ and $B, K X$ have properties $P_{n \rightarrow r}$ and $P_{m \rightarrow r}$ respectively. In addition we have this theorem.

TheOREM 3. If, under the conditions of Theorem 1 , the pair of matrices $A, X K$ has property $P$ (or property $L, c f$. Motzkin and Taussky (4)), then so has the pair $B, K X$, and conversely.

Proof. We need only remark that with the hypothesis of Theorem 3 we have $\theta(\lambda)=\prod_{i=1}^{r}\left(\lambda-f\left(\epsilon_{i}, \gamma_{i}\right)\right)$ for any polynomial $f(x, y)$ (or, in the case of property $L$, for $f(x, y)=\mu x+\nu y)$, where $\lambda^{n-r} \prod_{i=1}^{r}\left(\lambda-\gamma_{i}\right)$ is the characteristic polynomial of $X K$.

4. In this section we shall show how various known results are obtained from our theorems. Let $f(x, y)=x$. Then Theorem 1 reduces to the result that $A X=X B$ implies that the characteristic polynomials of $A$ and $B$ have a common factor of degree $r$, where $r$ is the rank of $X$. Next, set $f(x, y)=y$. Then $f_{0}(A)$ and $f_{0}(B)$ are zero, so that our theorem furnishes yet another proof that the characteristic polynomials of $X K$ and $K X$ differ by the factor $\lambda^{n-m}$. It is easy to see that the same is true for any polynomial $f(x, y)$, each of whose terms contain $y$. Finally, let $r=m$ and let $X$ consist of characteristic column vectors of $A$. Then $B$ is diagonal, in Theorem $2,\left(\beta_{r+1}, \ldots, \beta_{m}\right)$ is empty, and the result of Goddard (2) quoted in $\$ 1$ follows in a slightly stronger form on putting $f(x, y)=x+y$ in Theorem 2. If $m=n$ and $B=0$, so that $A X=0$, and if $f(x, y)$ is a polynomial without constant term, then the corollary to Theorem 1 becomes, with $K=I_{n}$,

$$
\left|\lambda I_{n}-f(A, X)\right| \lambda^{n}=\left|\lambda I_{n}-f(A, 0)\right|\left|\lambda I_{n}-f(0, X)\right|,
$$

which is a result proved elsewhere by Schneider (6).

Note added in proof. We have noticed that Theorem 1 of a paper just published by Miss Hazel Perfect (Duke math.' J. 22 (1955), 305-11) is a special case of the corollary to Theorem 1 above, namely, where $\lambda=0, f(x, y)=x-y$ and $X$ is of full rank.

\section{REFERENCES}

(1) Brader, A. Limits for the characteristic roots of a matrix, IV. Duke math. J. 19 (1952), 75-91.

(2) GoDdard, L. S. Proceedings of the International Mathematical Congress, Amsterdam (September 1954), Vol. II, p. 22.

(3) McCoy, N. H. On the characteristic roots of matrix polynomials. Bull. Amer. math. Soc. (1936), 592-600.

(4) Motzkrn, T. S. and Taussky, O. Pairs of matrices with property L. Trans. Amer. math. Soc. 73 (1952), 108-14.

(5) Perfict, H. Methods of constructing certain stochastic matrices. Duke math. J. 20 (1953), $395-404$.

(6) Schnemer, H. A pair of matrices with property $P$. Amer. math. Mon. 62 (1955), 247-9. 